\title{
BMJ Open Head and neck cancer risk factors in India: protocol for systematic review and meta-analysis
}

\author{
Aayush Poddar, ${ }^{1}$ Ritchlynn Ronald Aranha, ${ }^{1}$ Gothandam K Muthukaliannan, ${ }^{1}$ \\ Ramesh Nachimuthu, ${ }^{1}$ Rama Jayaraj ${ }^{2}$
}

To cite: Poddar A, Aranha RR, $\mathrm{K}$ Muthukaliannan $\mathrm{G}$, et al. Head and neck cancer risk factors in India: protocol for systematic review and meta-analysis. BMJ Open 2018;8:e020014. doi:10.1136/ bmjopen-2017-020014

- Prepublication history for this paper is available online. To view these files, please visit the journal online (http://dx.doi. org/10.1136/bmjopen-2017020014).

Received 11 0ctober 2017 Revised 21 July 2018 Accepted 26 July 2018

A Check for updates

(c) Author(s) (or their employer(s)) 2018. Re-use permitted under CC BY-NC. No commercial re-use. See rights and permissions. Published by BMJ.

${ }^{1}$ School of Bio Sciences and Technology, Vellore Institute of Technology, Vellore, Tamil Nadu, India

${ }^{2}$ College of Health and Human Sciences, Charles Darwin University, Darwin, Northern Territory, Australia

Correspondence to

Dr. Rama Jayaraj;

Rama.Jayaraj@cdu.edu.au

\section{ABSTRACT}

Introduction Demographic, behavioural and environmental factors have been associated with increased risk of head and neck cancer (HNC). We will review published reports and explore connections between risk factors and HNC incidence. This protocol aims to provide strategies for a systematic review and meta-analysis of HNC risk factor analysis in India. It also provides guidelines in order to visualise obtained HNC risk factor data in the form of a heat-map highlighting variations across gender, age and geographical location.

Methods and analysis We will identify well-established HNC risk factors and perform a comprehensive systematic review and meta-analysis to quantify each risk factor's impact on HNC incidence. A systematic search will be performed to identify the studies and published reports of HNC risk factors in India. Meta-analysis will be conducted to estimate the proportional contribution of the most prevalent risk factor in HNC on a city-wide basis in Indian states and territories.

Ethics and dissemination The review protocol draws on publicly available anonymised data without directly involving human participants and therefore requires neither formal human ethical review nor approval by a human research ethics committee. We published an outline of the protocol in the International Prospective Register of Systematic Reviews (PROSPER0) in 2017. The results will provide an updated analysis of HNC risk factor prevalence in India, and we will discuss the applicability of rehabilitation care. We plan to disseminate the findings of this systematic review through publication in a peerreviewed journal and presentation at relevant conference proceedings.

PROSPERO registration number CRD42017077758.

\section{INTRODUCTION}

The systematic review will generate up-todate information on the role of different risk factors of head and neck cancer (HNC) incidence in India. This study will provide the city-specific prevalence of $\mathrm{HNC}$ risk factors which may have implications on health policies for management of HNC and for establishing cancer care in profoundly affected areas.

\section{Strengths and limitations of this study}

- The study attempts to calculate the OR of head and neck cancer (HNC) occurrence due to risk factor prevalence while following geographical demarcation based on cultural adaptation.

- The study can be expanded into a time-trends analysis to analyse variations in an OR of HNC occurrence.

- Given the lack of infrastructure and funding in India, there is a possibility that the study participant population will not be representative of the overall population.

- The study primarily focuses on published papers as no such national or state-wide cancer registry exists which provides information on risk factor prevalence and associated HNC incidence.

The worldwide HNC trends for risk factor patterns have drastically changed in the past 15 years. ${ }^{1}$ It is considered as a lethal disease for approximately half of all diagnosed cases, owing to low awareness and late detection at advanced stages of cancer. ${ }^{2} \mathrm{HNC}$ is the third most common in India with 52067 deaths and 77003 cases diagnosed in $2012 .^{3}$ The real incidence is much more than the actual estimates as many cases of HNC go undiagnosed or unreported. Numerous reports highlight that risk factors are not only aetiological determinants of HNC but are also connected with increased risk of HNC prevalence. ${ }^{4-6}$ Previously published studies have demonstrated that alcohol consumption and tobacco use are the most significant risk factors of HNC in addition to HPV. ${ }^{7-9}$

The significant risk factors for HNC have already been elucidated. ${ }^{10}$ However, the likelihood chances of an individual developing HNC has not been studied thoroughly. This is due to the scarcity of published review papers in this context. This study will provide guidelines to help clinicians and scientists better understand the link between HNC and its risk factors, mainly smoking, alcohol 
consumption, HPV and betel-quid chewing in Indian patients with HNC.

The most significant risk factors are strongly associated with the sociocultural diversity and customs of India, ${ }^{11} 12$ and this obstacle leads to poor clinical outcomes. The connections between diverse risk factors including alcohol, HPV, tobacco smoking and tobacco chewing, significantly vary due to diverse demographic and lifestyle habits of people in India. ${ }^{13} 14$

\section{RATIONALE}

\section{What is the issue?}

There is a scarcity of quantitative analysis and data synthesis of the causal relationships between HNC and their risk factors in the Indian demography. This could be due to the lack of data linkage and data reporting of HNC incidence in addition to the absence of integrated national and state-wide functional cancer registry. The prevalence of $\mathrm{HNC}$ is frequently dissimilar in different states and communities of the Indian population. It differs significantly from one community to another, and varies across various cities within the same geographical location, primarily depending on the practices and lifestyles of the people in that location. ${ }^{15}$ Furthermore, there are several factors associated with an increased risk of HNC such as diverse demographical, socioeconomic, clinicoepidemiological, clinicopathological and biological characteristics of Indian patients with HNC that will benefit the study in understanding the precise difference between these factors.

\section{How will our study address this?}

This study will be the first of its kind to use meta-analysis in the evaluation of HNC risk factors in 29 Indian states and 7 union territories. The meta-analysis offers an accurate degree of consistency by quantifying the extent of the variation compared with narrative synthesis. Quantitative synthesis will allow enumerating the diverse roles of the published risk factors of HNC to develop an HNC risk prediction model for future clinical research in India. The pooled effect size of HNC risk factors and the relative weight to the overall meta-analysis of the published studies from diverse Indian states and territories can contribute to achieving the precision model to assess the specific dose-response association between multilevel risk factors and risk of HNC.

\section{How will it help?}

Since India is cosmopolitan in culture, while being quite economically and sociodemographically distinct from other Western countries, our findings will also be useful in further research for developing risk prediction models of HNC. This proposed systematic review and meta-analysis protocol will provide comprehensive and up-to-date information on the different combinations of risk factor relationship with HNC. This will also identify more appropriate HNC risk factor reports and studies published in this context. This extracted data will aid in filling the knowledge gaps of HNC risk factor distribution in 29 states and 7 union territories of India. The effect size estimates of risk factor distribution will help to address the research priorities identified by WHO and National Centre for Disease Information and Research (NCDIR) National Cancer Registry Programme (NCRP) initiated by Indian Council of Medical Research (ICMR). This protocol outlines the strategies for a systematic review and meta-analysis that could be helpful to Indian oral health and care, public health and political actions leading to personalising interventions for individuals at risk of HNC. This protocol provides in-depth information on $\mathrm{HNC}$ with the study objectives and design, search strategies, eligibility criteria, data extraction and synthesis, that is most appropriate to cancer researchers, clinicians and epidemiologists. This systematic review and meta-analysis will prospectively help in improving the early detection by addressing the percentage of prevalence and geographical distribution of risk factors in addition to early screening and treatment facilities thereby creating awareness among the high-risk Indian population. These public health measures will have an impact on reducing HNC mortality in India.

This protocol aims to describe the methodological approach for conducting systematic review and meta-analysis on risk factor distribution of $\mathrm{HNC}$ in the Indian demography. Given the potential importance of this study, the systematic review and meta-analysis are to quantify $\mathrm{HNC}$ incidence in association with risk factor prevalence in different Indian cities. The subgroup analysis with varying combinations of risk factors would further aid in figuring out the likelihood of developing HNC on a city-specific scale and predicting the endemic high-risk zones.

\section{METHODS \\ Study design and participants}

The authors will consider reports and also all published studies as well as unpublished studies from conference proceedings. The anticipated date of commencement of literature search for identifying studies is 15 July 2018 and the anticipate date of completion is 15 December 2018. The study will include all studies that have clearly defined HNC risk factors expressed both individually and in combinations. Authors will also include studies describing the general human population in different geographical regions of India diagnosed with laboratory and clinically confirmed HNC from all ethnicities and socioeconomic backgrounds.

There will be no limits on study participants in terms of: a. Demographic parameters such as age, gender, ethnicity and employment.

b. Clinicopathological parameters such as anatomical sites, tumour stage, nodal status, nodal stage, postoperative radiotherapy, histological grade. 
c. Clinical outcomes such as recurrence (local and regional) and patients' survival such as overall survival (OS), disease-free survival (DFS) and disease-specific survival (DSS).

Authors will include risk factor studies pertaining to incidence, prevalence and mortality of HNC in India. These studies will be carried out independently and will not be based on any global or national cancer registry for the statistical data of HNC risk factor distribution. Studies will be selected according to the criteria outlined below.

\section{Study selection criteria}

\section{Inclusion criteria}

- The HNC risk factor study has performed independent data extraction and has not relied on any state, national or global cancer registries.

- Study provides statistical data regarding the risk factor associated with HNC incidence in India.

- Study talks about the city-wise risk factor prevalence within India.

- The inclusion of factor based on the strength of the factor and the availability of at least three levels of interactions such as dose, exposure and level of associated risk.

- Language: English.

\section{Exclusion criteria}

- The study has stated HNC screening.

- The study uses different HNC in-vitro analysis and evaluations.

- Review articles and studies comparing the different genetic profiles in HNC.

\section{Selection criteria for participants}

\section{Inclusion criteria}

1. Participants of any age with $\mathrm{HNC}$ or receiving HNC treatment will be considered.

2. Participants with a clearly confirmed diagnosis of HNC.

3. Participants based in India.

\section{Exclusion criteria}

1. Participants' age or age range not clearly mentioned.

2. Study participants' confirmative diagnoses of HNC have not been clearly identified.

3. Self-reporting of the disease and questionable survey and screening methods of deduction have been employed.

\section{Setting}

There will be no restrictions by type of clinical setting, and authors will include studies at all levels of healthcare setting (such as primary, secondary and tertiary healthcare) and those conducted in the community.

\section{Language}

Authors will include articles reported in English language.

\section{Information sources}

The authors will develop a comprehensive literature search strategy using Medical Subject Headings (MeSH) and text words related to the prevalence of HNC risk factors in India. The authors will scan the reference list through Cochrane Library, Embase, MEDLINE, PubMed, Science Direct, Scopus and Web of Science. The authors will also search multiple electronic bibliographic databases to identify the grey literature and unpublished studies from conference proceedings. The authors will circulate the bibliography of the included articles to the systematic review team.

\section{Searching other resources}

The major metropolitan city and hospital-based cancer registries in 29 states and 7 union territories of India will be integrated with the following reports by national and international cancer registries:

- Cancer Incidence in Five Continents by WHO: International Agency for Research on Cancer (IARC).

- Global Cancer Observatory by WHO: IARC.

- GLOBOCAN 2012 by WHO: IARC.

- Global Heath Estimate 2012 by WHO: Department of Health Statistics and Information Systems.

- Three-Year Report of Population-Based Cancer Registries 2012-2014 by NCDIR-NCRP initiated by ICMR.

\section{Search strategy}

The systematic review and meta-analysis team will consider both qualitative and quantitative HNC risk factor studies primarily focusing on the Indian demography. All authors will provide their inputs for the draft Scopus search strategy to ensure that it retrieves a high proportion of eligible studies. After the Scopus strategy is finalised, it will be adapted to the syntax and subject headings of the other electronic bibliographical databases to be searched. The specific search strategies will be created by all authors after consultation with the review team.

Draft Scopus search:

1. 'Head and Neck Cancer' [Topic] AND 'India' [Topic].

2. 'Head and Neck Cancer' [Topic] AND 'Risk Factors' [Topic] AND 'India' [Topic].

3. 'Head and Neck Cancer' [Topic] AND 'Risk Factors' [Topic] AND 'India' [Topic] AND 'Geographical incidence' [Topic].

4. 'Head and Neck Cancer' [Topic] AND 'Alcohol' [Topic] AND 'India' [Topic].

5. 'Head and Neck Cancer' [Topic] AND 'Smoking' [Topic] AND 'India' [Topic].

6. 'Head and Neck Cancer' [Topic] AND 'Betel' [Topic] AND 'India' [Topic].

7. 'Head and Neck Cancer' [Topic] AND 'HPV' [Topic] AND 'India' [Topic].

\section{Study records}

Data management

The HNC risk factor literature will be fed into a reference management software, EndNote. This will contribute to 
a strong working relationship among the review team during the study selection process. The reviewers will select the studies based on selection criteria and will upload relevant studies into EndNote. This will yield a Preferred Reporting Items for Systematic Reviews and Meta-Analyses flow diagram after the screening process by the HNC risk factor review team. HNC reviewers will also be using the traditional forms of data management in this process. Authors will avoid duplications when compiling together from multiple reports of the same study by including study design, HNC participants' characteristics and risk factor associations. The corresponding authors will be contacted for missing information in the studies.

\section{Selection process}

The author team will review the titles and abstracts related to HNC risk factors in India. They will obtain the full length of all titles that meet the selection criteria. Authors will screen the full-length articles and confirm whether the screened articles meet the selection criteria.

\section{Data collection process}

The references extracted from the full-length articles will be reviewed to identify other publications of interest. References cited in the retrieved, as well as selected publications, will be considered to find additional articles in this context. The HNC risk factor data extraction form will be created and used by the review team during the data collection process. This particular form will be piloted on randomly selected eligible studies of HNC risk factors. Any discrepancies between the two groups will be sorted out via mutual discussion.

\section{Data items}

Authors will extract the various parameters using the HNC risk factor data extraction form. The key data items include:

a. Characteristics of studies (including author, year of publication, a geographical region within India that the study talks about, the year when the study took place and type of studies such as cross-sectional studies, observational studies and longitudinal studies).

b. Characteristics of the study participants consist of three classifications: HNC participants' demographic characteristics (such as age, gender, ethnicity and employment).

c. Clinicopathological characteristics (such as anatomical sites, tumour stage, nodal status, nodal stage, postoperative radiotherapy and histological grade).

d. Clinical outcomes (such as recurrence (local and regional).

e. Patients' survival such as OS, DFS and DSS).

f. Characteristics of individual HNC risk factors (such as alcohol consumption, tobacco smoking, HPV and betel-quid chewing) and their combinations (such as alcohol and HPV, and tobacco smoking and HPV, and tobacco chewing and HPV). g. Prevalence of HNC risk factors in different cities in India and its associations with $\mathrm{HNC}$ incidence.

\section{Outcomes}

Primary outcome

The primary outcome is to evaluate the risk factor prevalence and its associations with HNC in India.

\section{Secondary outcome}

The secondary outcome is to link the variations in HNC risk factors with different geographical locations in India in addition to other demographical, clinicopathological and clinical parameters.

\section{Risk of bias in individual studies}

The authors will collect the risk factor information from individual studies during their data synthesis phase using defined procedures for possible risk of bias. The defined procedures will include study validity based on specific parameters such as a number of patients with HNC, year of publication, mention of International Classification of Diseases (ICD) code, disease diagnosis and confirmation, study locations and study period. The review team will decide on possible risk of bias within the extracted information from the included studies, either high risk or low risk. Two authors will independently make these decisions, and disagreements will be resolved by team decision and consultation with the third author. The studies will be assessed for risk of bias using guideline formulated by Effective Health Care Program, ${ }^{15}$ and we will also use Newcastle-Ottawa Scale ${ }^{16}$ for the methodological assessment of cohort studies.

\section{Data synthesis}

Authors will describe the risk factor prevalence with reference to ICD code for HNC (lip and oral (C00-08), nasopharynx (C10), other pharynx (C09-10, C12-14) and larynx (C32)). The authors will also include different clinical studies with the different combination of risk factors and different age ranges and studies with varying times of follow-up. This process will be performed in two phases. The first phase consists of identification and dissemination of risk factor resources collected, followed by critical study and participant data items extracted. The second phase will focus on utilisation of retrieved data items to estimate the survival trends among the HNC participants using Comprehensive Meta-Analysis Software. The software analysis will yield the information about the heterogeneity of OR using Cochran's $\mathrm{Q}$ test and Higgins' ( $\mathrm{I}^{2}$ statistic. ${ }^{17}$ Heterogeneity between the HNC risk factor studies will be assessed using the $\mathrm{I}^{2}$ statistic, wherein substantial heterogeneity would be indicated by obtaining an $\mathrm{I}^{2}$ value greater than $50 \%$. Fixed or random effects model will be applied depending on the heterogeneity. $Q$ test statistical significance will be considered at a $p$ value of $<0.01$. Publication bias will be assessed using Harbord-Egger's bias indicator test, ${ }^{18}$ Orwin's classic fail-safe $\mathrm{N}$ test, ${ }^{19} \mathrm{Begg}$ and Mazumdar's rank correlation 
test ${ }^{20}$ Duval and Tweedie's trim and fill calculation ${ }^{21}$ and inverted funnel plot.

\section{Subgroup analysis and meta-regression model}

Subgroup analysis will be performed on primary outcomes with subgroups defined by different study locations throughout India of reported incidence. Different combinations of the HNC risk factors and its associations with HNC incidence and prevalence will be measured. The source of heterogeneity will be assessed using meta-regression analysis of fitting covariables. Heterogeneity will be considered significant if $p$ value is $<0.05$. The heterogeneity of proportional contributions of risk factor associations with one or more study variable will be assessed using meta-regression analysis. The impact of proportional contributions of risk factor and combination of risk factors on fitting covariables, including gender distribution, methods of data collection, sample size, research quality and sampling procedure, will be calculated using meta-regression model. It needs a large ratio of studies for assessing the impact of combinations of risk factors to calculate true regression.

\section{Patient and public involvement}

No patients will be involved in this study.

\section{Ethics and dissemination}

We plan to publish the results of this systematic review and meta-analysis in a peer-reviewed journal and present at relevant conference proceedings.

\section{DISCUSSION}

The precise risk-factor analysis with respect to HNC incidence cannot be sufficiently explained in the published studies. Most published clinical studies focus on major referral centres, or city-wise or state-wise HNC incidence and prevalence. ${ }^{22}{ }^{23}$ Estimation of a national risk factor prevalence is an urgently needed agenda from the perspective of epidemiologists to identify low-risk and high-risk endemic zones. ${ }^{24}$ Further evaluations apart from our defined scope of this study are not advisable. Structuring a systematic review and meta-analysis around the framework of a registered protocol will offer a more consistent strategy. ${ }^{25}$ Furthermore, a reviewed protocol will allow more in-depth analysis. HNC incidence is on a staggering rise. ${ }^{26-29}$ A large portion of this increase is attributed to adults who indulge in multifarious HNC risk factors widely prevalent in India. ${ }^{2430}$ Immediate introductions of control measures would be a proactive step in order to curb the rising HNC incidence. ${ }^{31} 32$

Contributors RJ conceived this study and provided supervision and mentorship to AP and RRA. RJ and AP led the development of the study protocol and design, wrote the first draft of the protocol, and coordinated and integrated comments from coauthors. RJ, GKM, NR and AP critically revised and edited successive drafts of the manuscript and gave input to the final draft of the protocol. RJ provided methodological guidance on the overall development of the protocol. All authors read and approved the final version of the manuscript.
Funding The authors have not declared a specific grant for this research from any funding agency in the public, commercial or not-for-profit sectors.

Disclaimer Neither the authors' institutions nor any funder or sponsor played a role in developing the protocol. The authors wrote this protocol during their routine work in their respective institutions, but the views expressed therein are those of the authors and not those of their institutions.

Competing interests None declared.

Patient consent Not required.

Ethics approval The study does not require formal ethics approval by a human research ethics committee because this review protocol collects risk factor data from publicly published reports and studies.

Provenance and peer review Not commissioned; externally peer reviewed.

Open access This is an open access article distributed in accordance with the Creative Commons Attribution Non Commercial (CC BY-NC 4.0) license, which permits others to distribute, remix, adapt, build upon this work non-commercially, and license their derivative works on different terms, provided the original work is properly cited, appropriate credit is given, any changes made indicated, and the use is non-commercial. See: http://creativecommons.org/licenses/by-nc/4.0/.

\section{REFERENCES}

1. Johnson-Obaseki S, McDonald JT, Corsten M, et al. Head and neck cancer in Canada: trends 1992 to 2007. Otolaryngol Head Neck Surg 2012;147:74-8.

2. Warnakulasuriya S. Global epidemiology of oral and oropharyngeal cancer. Oral Oncol 2009;45(4-5):309-16.

3. GLOBOCAN. Estimated Cancer Incidence, Mortality and Prevalence Worldwide in 2012: World Health Organization: International Agency for Research on Cancer, 2012.

4. Döbrossy L. Epidemiology of head and neck cancer: magnitude of the problem. Cancer Metastasis Rev 2005;24:9-17.

5. Ragin CC, Modugno F, Gollin SM. The epidemiology and risk factors of head and neck cancer: a focus on human papillomavirus. J Dent Res 2007;86:104-14

6. Sturgis EM, Wei Q, Spitz MR. Descriptive epidemiology and risk factors for head and neck cancer. Semin Oncol 2004;31:726-33.

7. Blot WJ, McLaughlin JK, Winn DM, et al. Smoking and drinking in relation to oral and pharyngeal cancer. Cancer Res 1988;48:3282.

8. Hashibe M, Brennan P, Benhamou S, et al. Alcohol drinking in never users of tobacco, cigarette smoking in never drinkers, and the risk of head and neck cancer: pooled analysis in the International Head and Neck Cancer Epidemiology Consortium. J Natl Cancer Inst 2007;99:777-89.

9. Franceschi S, Talamini R, Barra S, et al. Smoking and drinking in relation to cancers of the oral cavity, pharynx, larynx, and esophagus in northern Italy. Cancer Res 1990;50:6502.

10. Singh J, Ramamoorthi R, Baxi S, et al. The risk factors of head and neck cancer and their general patterns in Australia: a descriptive review and update. J Environ Pathol Toxicol Oncol 2014;33:45-57.

11. Addala L, Pentapati CK, Reddy Thavanati PK, et al. Risk factor profiles of head and neck cancer patients of Andhra Pradesh, India. Indian J Cancer 2012;49:215-9.

12. Sharan RN, Mehrotra R, Choudhury $Y$, et al. Association of betel nut with carcinogenesis: revisit with a clinical perspective. PLoS One 2012; 7:e42759.

13. Hashibe M, Brennan P, Chuang SC, et al. Interaction between tobacco and alcohol use and the risk of head and neck cancer: pooled analysis in the international head and neck cancer epidemiology consortium. Cancer Epidemiol Biomarkers Prev 2009;18:541-50.

14. Vargas-Ferreira F, Nedel F, Etges A, et al. Etiologic factors associated with oral squamous cell carcinoma in non-smokers and nonalcoholic drinkers: a brief approach. Braz Dent J 2012;23:586-90.

15. Viswanathan M, Ansari MT, Berkman ND, et al. Assessing the risk of bias of individual studies in systematic reviews of health care interventions, 2012.

16. Wells G, Shea B, O'connell D, et al. The Newcastle-Ottawa Scale (NOS) for assessing the quality of nonrandomised studies in metaanalyses. Ottawa (ON): Ottawa Hospital Research Institute, 2009. Available in March 2016

17. Higgins JP, Thompson SG. Quantifying heterogeneity in a metaanalysis. Stat Med 2002;21:1539-58.

18. Egger M, Davey Smith G, Schneider M, et al. Bias in meta-analysis detected by a simple, graphical test. BMJ 1997;315:629-34. 
19. Orwin RG. A fail-safeN for effect size in meta-analysis. Journal of Educational Statistics 2016;8:157-9.

20. Begg CB, Mazumdar M. Operating characteristics of a rank correlation test for publication bias. Biometrics 1994:50:1088

21. Duval S, Tweedie R, Trim TR. Trim and fill: A simple funnel-plot-based method of testing and adjusting for publication bias in meta-analysis. Biometrics 2000;56:455-63.

22. Bhattacharjee A, Chakraborty A, Purkaystha P. Prevalence of head and neck cancers in the north east-An institutional study. Indian J Otolaryngol Head Neck Surg 2006;58:15-19.

23. Sharma P, Saxena S, Aggarwal P. Trends in the epidemiology of oral squamous cell carcinoma in Western UP: an institutional study. Indian J Dent Res 2010;21:316-9.

24. Siddiqui MS. Chandra R Fau - Aziz A, Aziz A Fau - Suman S, et al. Epidemiology and histopathological spectrum of head and neck cancers in Bihar, a state of Eastern India. 2476-762x.

25. Shamseer L, Moher D, Clarke M, et al. Preferred reporting items for systematic review and meta-analysis protocols (PRISMA-P) 2015: elaboration and explanation. BMJ 2015;350:g7647.

26. Three-Year Report of Population Based Cancer Registries 2012-2014. Incidence, Distribution, Trends in Incidence Rates and Projections of Burden of Cancer (Report of 27 PBCRs in India). Three-Year Report of Population Based Cancer Registries. Bangalore: National Cancer Registry Programme (NCRP) - National Centre for Disease Informatics \& Research (NCDIR) by Indian Council of Medical Research (ICMR), 2016.

27. Cancer Incidence in Five Continents. World Health Organization (WHO): International Agency for Research on Cancer (IARC), 2002; VIII.

28. Jemal A, Bray F, Center MM, et al. Global cancer statistics. CA Cancer J Clin 2011;61:69-90.

29. World Cancer Report. World Health Organization (WHO): International Agency for Research on Cancer (IARC), 20142014

30. Simard EP, Torre LA, Jemal A. International trends in head and neck cancer incidence rates: differences by country, sex and anatomic site. Oral Oncol 2014;50:387-403.

31. Patel UA, Brennan TE. Disparities in head and neck cancer: assessing delay in treatment initiation. Laryngoscope 2012;122:1756-60.

32. Krishnatreya M, Kataki AC, Sharma JD, et al. Educational levels and delays in start of treatment for head and neck cancers in North-East India. Asian Pac J Cancer Prev 2014;15:10867-9. 